\title{
Inversion Analysis of Seepage Parameters Based on Improved Shuffled Frog Leaping Algorithm
}

\author{
Li Qun Xu $\mathbb{D}^{1}$ and Ling $\operatorname{Li} \mathbb{D}^{1,2}$ \\ ${ }^{1}$ School of Water Conservancy and Hydropower, Hohai University, Nanjing 210098, China \\ ${ }^{2}$ Ningbo Water Conservancy \& Hydropower Planning and Design Institute Co., Ltd., Ningbo 315192, China \\ Correspondence should be addressed to Ling Li; 553261311@qq.com
}

Received 22 June 2020; Revised 12 September 2021; Accepted 14 September 2021; Published 12 October 2021

Academic Editor: Bartlomiej Blachowski

Copyright ( $) 2021 \mathrm{Li}$ Qun Xu and Ling Li. This is an open access article distributed under the Creative Commons Attribution License, which permits unrestricted use, distribution, and reproduction in any medium, provided the original work is properly cited.

\begin{abstract}
Aiming at overcoming the defects such as slow searching speed and easily trapping into local extremum at anaphase of the shuffled frog leaping algorithm (SFLA), based on the Evolutionary Exploration strategy, a more effective shuffled frog leaping algorithm, Improved Shuffled Frog Leaping Algorithm (ISFLA), which can be applied to the inverse analysis of seepage parameters to dams, is proposed. With the introduction of the threshold value selection in the local search of the original initial population to improve the best frogs in memeplex, the improved algorithm overcomes the shortcomings of traditional SFLA which can easily fall into a local optimum. By comparative analysis between the laboratory test and numerical simulation, the effectiveness and accuracy of ISFLA are demonstrated by the application to the inversion analysis of seepage parameters of earth dams. Furthermore, the inversion analysis of seepage parameters to the earth dam in Lianyungang China is studied by the ISFLA. Moreover, the seepage characteristics of the dam are evaluated; thus, the suggestion that the dam should be reinforced is put forward. All the results show that ISFLA in an inverse analysis of seepage parameters of dams has excellent value to hydropower engineering.
\end{abstract}

\section{Introduction}

During the long operation time, dams and their foundations suffered from various loads, which may lead to huge changes in the seepage parameters of the structure. Meanwhile, the leakage problem is one of the main problems of the dangerous earth-rock dams [1]; to ensure dams' safety operation, the real running conditions of the dams should be known through safety monitoring [2]. The seepage characteristic analysis of the dams is the most important way to realize the safety monitoring of the dam [3]. There are two methods to analyze the seepage characteristic: one is the direct analysis and the other is the inverse analysis. The direct analysis is based on the establishment of dams' monitoring mathematical model by dams' observation data. The key of this method is to evaluate the seepage parameters of dams and foundations correctly. Inverse analysis can revise seepage parameters of dams and rock foundations based on dams' monitoring information, so it can make the forecasting value and monitoring value more accordant; therefore, it can provide more objective material parameters for the establishment of dams' security monitoring mathematical model. The recent development of information technology created the premise of the constant progress of the numerical method. The inverse analysis method includes the analytic method, graphic method, and numerical method. In addition, the numerical method comprises a positive algorithm, opposite algorithm, statistics analysis method, and so on. The positive algorithm has the most obvious character; that is, the mature optimization method and efficient computer program are used, so it can be well used in resolving many kinds of inverse analysis problems with linear and nonlinear parameters. In the meantime, since this method can avoid solving an unknown value oppositely, it has the advantages of good applicability and being easy to put into practice. The optimization search model is a major part of the positive algorithm. Optimization has wide applicability; nowadays, neural networks 
and genetic algorithms have been applied in the inverse analysis of dams' parameters [4], and the genetic algorithm and Gauss-Newton method have been used in the inverse analysis of parameters identification of groundwater flow model [5] and solving the permeability coefficients of gravity dam foundation [6].

However, traditional optimization methods also have drawbacks, such as outcomes' relying on initial values, difficulty in multiple parameters optimization, and outcomes easily trapped into local extremum at anaphase [7]. To solve these problems, a lot of new optimization algorithms have been proposed [8-10]. The shuffled frog leaping algorithm (SFLA) is firstly raised by Eusuff and Lansey for the sake of solving group optimization problems in 2003 [11]. SFLA, which is a combination of the Memetic Algorithm (MA) with strong local search ability and Particle Swarm Optimization (PSO) with good global search capability, has some traits, such as simple concept, a few parameters, fast computing speed, powerful global optimization capability, and easy implementation [12-14]. After SFLA's being put forward, it gains wide attention from scholars, becomes a research hotspot in the area of evolution calculation rapidly, and has been put into use in various kinds of areas such as water resource management [15], civil engineering [16], and information engineering [17]. According to the evolution strategy of SFLA, only the worst frog is evolved, which is based on the local best frog or global best frog, during the local evolution in the memeplex; thus, it takes the defects of the slow searching speed and easily trapping into local extremum at anaphase [18]. Inspired by Zhu and Lin [19] analysis of the convergence of traditional SFLA, we introduced a threshold value selection in the local search of the original initial population to overcome the defects.

Based on the evolution strategy of SFLA, we propose an Improved Shuffled Frog Leaping Algorithm in this paper and apply it to the inverse analysis of dams' seepage parameters.. The effectiveness and accuracy of the ISFLA are assessed by the comparative analysis between the laboratory test and numerical simulation. Through the inversion analysis of seepage parameters to the earth dam in Lianyungang China, the effectiveness of the proposed algorithm for solving complex inversion problems in engineering seepage is verified. Moreover, the seepage characteristics of the dam are evaluated, thus the suggestion that the dam should be reinforced is put forward.

\section{Shuffled Frog Leaping Algorithm}

Shuffled Frog Leaping Algorithm (SFLA) is a new metaheuristic population evolutionary algorithm [20]. Based on imitating the process of frogs searching for foods, SFLA combines the global thoughts exchange and the local search. The local search can make local individuals deliver their thoughts, and the global thoughts exchange makes the exchange of thoughts between individuals possible. According to this algorithm, the group consists of a group of frogs that have the same structure, and every frog represents a feasible solution. The whole group is divided into several memeplexes, and then each memeplex is defined as a frog set with one thought different from others. The way that frogs in the memeplex do the local search for the solution is similar to PSO. When the times of the local search are equal to the defined times, frogs are remixed to do the global thoughts exchange. The process of global thoughts exchange and the local search continuously leapfrog until the results can meet the requirements of convergence. This balance strategy that is the major character of SFLA enables the algorithm to jump out of the local extremum in order to move towards the global extremum.

\subsection{Elements of SFLA}

2.1.1. Population. In SFLA, the population is an ordered frogs group that is randomly generated and separated throughout the wetland. Individual frog is a carrier of memetic information. Every piece of memetic information is made up of several information elements. Corresponding to the memetic information every frog carries, there is an adaptability value used for assessing the frogs' usefulness.

2.1.2. Memeplex. These frogs, sorted by their objective function value since the population has been generated, are equally divided into several independent memeplexes. Every memeplex has its own unique thoughts. According to the memetic evolution strategy, these frogs in every memeplex can do the local search in the solution space, while the information is transmitted among individual frogs.

2.1.3. Submemeplex. Aiming at preventing the situation of trapping into the local extremum at anaphase, the concept of submemeplex is introduced to SFLA. The better the adaptability value of these frogs in the memeplex, the greater chance to enter into a submemeplex. The submemeplex can replace the memeplex in doing the local search in the solution space. Additionally, the submemeplex is not invariant. When the local search has finished, frogs in the memeplex are remixed to do the global thoughts exchange. Then, these frogs are resorted by their objective function value to generate a new submemeplex. In this way, the adaptability value of these frogs can be ensured and the global extremum can be achieved.

2.2. Parameters of SFLA. There are 10 main parameters of SFLA; for example, $F$ is the number of frogs; $m$ is the number of memeplex, $n$ is the number of frogs in one memeplex, $S_{\max }$ is the maximum allowable step length, $P_{X}$ is the global best solution, $P_{B}$ is the local best solution, $P_{W}$ is the local worst solution, $q$ is the number of frogs in a submemeplex, $L S$ is the number of times of the local memetic evolution, SF is the number of times of the global thoughts exchange.

2.3. Algorithm Ending Conditions. There are two controlling conditions to end the running of SFLA. One is that the global best solution has achieved the required precision, and another one is that the global search has reached the maximum number of times defined in advance. When either of these 
above two conditions is satisfied, the algorithm will be ended which means the global extremum has been obtained.

2.4. Algorithm Flow. The algorithm includes global optimization and local optimization.

(A) Population are randomly generated by $F$ frogs. Each frog has a solution in the solution space, $\boldsymbol{X}=\left(x_{1}, x_{2}\right.$, $\left.x_{3}, \ldots, x_{s}\right)$. By the establishment of the objective function, the adaptability value of each frog is generated.

(B) After generating the initial population, the individuals in the frog population are sorted in descending order according to their adaptability value; thus, the global best solution $P_{X}$ is obtained. A total of $F$ frogs were divided into $m$ subpopulations. Define $M$ as the gather of frog memeplex, and the allocation process of $M_{k}$ can be described as follows:

$$
M_{k}=\left\{X_{k+m(l-1)} \in P \mid 1 \leq l \leq n\right\}, \quad 1 \leq k \leq m .
$$

(C) Optimize the local best solution of the populations.

(a) The population number is set to $i m=0$, using it to mark the population which currently has evolved. The independent evolution number is set to in $=0$. To judge whether the evolutionary steps have been completed, in is compared to the number of local evolution LS. During this time, the best and worst solutions have been searched for. The best solution in the memeplex is marked as $P_{B}$, and the worst solution is marked as $P_{W}$.

(b) Let $i m=i m+1$, and do a local search of the next memeplex.

(c) Let in $=i n+1$, and do an independent evolution of the next memeplex.

(d) Improve frogs in every memeplex. According to equations (2) and (3), the worst frog in the memeplex is evolved:

$$
\begin{aligned}
S & =\operatorname{Rand} \times\left(P_{B}-P_{W}\right), \\
\operatorname{New} P_{W} & =P_{W}+S, \quad S \in\left(-S_{\max }, S_{\max }\right),
\end{aligned}
$$

where $\operatorname{Rand}()$ is a random number in the range $[-1,1] . S$ is the step length of frog leaping, that is, the moving distance of the worst frog in memeplex. New $P_{W}$ is the local worst solution $P_{W}$ after evolution.

(e) During the evolution process, New $P_{W}$ should be the new $P_{W}$ if the worst frog has been evolved by step(d). Otherwise, $P_{B}$ in equation (2) is replaced by $P_{X}$ to update $P_{W}$. If the worst frog is still not evolved, the evolution is stopped; then, a new random frog is generated to replace $P_{W}$.

(f) Repeat this procedure until iteration times reach $L S$, which means the evolution of the memeplex is completed. Let in $=0$ and do the next step. (g) If $i m<m$, then go back to step (b). Otherwise, end local search step (C) and return to the global search step.

(D) After the allocation is completed, mix each memeplex. According to the following formula, $q$ individuals are randomly selected from each group to enter the subgroup and sorted in descending order according to the adaptability value, and the global optimal solution $P_{X}$ is recorded:

$$
P_{j}=\frac{2(n+1-j)}{[n(n+1)]}, \quad j \in(1, n),
$$

where $j$ is the $j^{\text {th }}$ frog, $P_{j}$ is the probability of the $j^{\text {th }}$ frog being chosen. If the scale of the memeplex is not very large, this step can be omitted.

(E) When the depth search is completed for all communities, if the number of global hybrid iterations is satisfied, the evolutionary process ends and the optimal solution is output. Otherwise, the whole frog population is remixed and rearrange by their objective function value. Then return to step (b) and repeat the previous steps until any stop criterion is met.

The implementation of the above algorithm is shown in Figure 1.

\section{Improved Shuffled Frog Leaping Algorithm (ISFLA)}

According to the evolution strategy of SFLA, only the worst frog is evolved, which is based on the local best frog or global best frog, during the local evolution in the memeplex. Thus, it takes the defects of the slow searching speed and easy trapping into the local extremum at anaphase. This is because the spatial location of the algorithm will change greatly when updating $P_{W}$. When the dimension of variables increases, the change becomes larger, which increases the number of iterations and greatly increases the length of calculation.

An Improved Shuffled Frog Leaping Algorithm (ISFLA) is proposed to overcome these defects. Threshold value selection is introduced in the local search of the original initial population. According to equations (2) and (3), random discriminant conditions are added to $P_{W}$. When the random number is larger than the threshold value, the frog jump step length on the dimension solution is still following equation (2), and when the random number is smaller than the threshold value, let $S=0$. This method can reduce the step gap and is more conducive to the convergence of iteration.

The worst frog improvement after optimization can be expressed as follows:

$$
\text { When } \begin{aligned}
R \geq R_{0} S & =\text { Rand } \times\left(P_{B}-P_{W}\right) \\
\text { or } S & =0, \\
\operatorname{New} P_{W} & =P_{W}+S, \quad S \in\left(-S_{\text {max }}, S_{\text {max }}\right),
\end{aligned}
$$


where $R_{0}$ is a local threshold and $R$ is a random number in the range $[0,1]$.

To further reduce the blindness of local worst frog improvement and improve the operation efficiency, different local update thresholds $R_{0}$ can be used according to the numerical range in the dimensional solution space. If the range of component values is large, a smaller update probability is used to increase the threshold value; if the range of component values is small, a larger update probability is used to reduce the threshold value.

To test the effectiveness of the improved algorithm, the following test functions are used to test the SFLA and its improved algorithm [4].

(1) Sphere

$$
f_{1}(x)=\sum_{i=1}^{N} x_{i}^{2}, \quad x_{i} \in[-5.12,5.12] .
$$

(2) Rosenbrock

$$
f_{2}(x)=\sum_{i=1}^{N-1}\left[100 \times\left(x_{i+1}-x_{i}^{2}\right)+\left(x_{i}-1\right)^{2}\right], \quad x_{i} \in[-5,5] .
$$

(3) Griewangk

$$
\begin{aligned}
f_{3}(x)= & 1+\sum_{i=1}^{N} \frac{x_{i}^{2}}{4000} \\
& -\prod_{i=1}^{n}\left(\cos \frac{x_{i}}{\sqrt{i}}\right), \quad x_{i} \in[-600,600] .
\end{aligned}
$$

(4) Schaffer's f7

$$
\begin{array}{r}
f_{4}(x)=\sum_{i=1}^{N-1}\left(x_{i}^{2}+x_{i+1}^{2}\right)^{0.25}\left[\sin ^{2}\left(50\left(x_{i}^{2}+x_{i+1}^{2}\right)^{0.1}\right)+1\right], \\
x_{i} \in[-100,100] .
\end{array}
$$

Among them, equation (6) is a relatively simple singlemode function. Equation (7) is a classical complex optimization problem function. It is often used to evaluate the efficiency of the optimization algorithm. Equations (8) and (9) are standard multimode functions with multiple local extremum points, which easily make the algorithm fall into a local optimal solution.

Each test function is run 50 times independently, where $F=200, m=20$, and $L S=10$. Do 500 times of the global thoughts exchange, and set the local update threshold $R_{0}=0.6$. The results are as shown in Table 1 .

It can be seen from Table 1 that, for the above four test functions, the optimal solution of the improved SFLA algorithm is close to the theoretical optimal solution. Moreover, for the relatively complex Rosenbrock function and Chaffer's F7 function, the improved SFLA can solve the problem effectively under the same number of mixed iterations, while the basic SFLA solution is easy to fall into the local optimum problem. That proves the improved SFLA method is effective.

\section{Procedures of Inverse Analysis of Dams' Seepage Parameters}

(a) The finite element model for seepage analysis is established based on the geological investigation data, design data, and monitoring data.

(b) The seepage parameters of dams and their foundations are solved as frogs $\left(k_{1}, k_{2}, \ldots, k_{n}\right)$ in waters, the number of frogs is $F$, the number of memeplexes is $m$, the number of frogs in one memeplex is $n$, the maximum allowable step length is $S_{\max }$. The initial value of these frogs is generated as the preliminarily ascertain seepage parameters which are based on the investigation.

(c) According to these seepage parameters, the seepage characteristics, such as the value of the waterhead $\left(h_{1}, h_{2}, \ldots, h_{m}\right)$ and seepage flow $\left(Q_{i}\right)$ that can be calculated by the finite element programming for seepage analysis. The square sum of the difference between the value of these above-calculated seepage characteristics and the value of monitoring value can be defined as the objective function, that is,

$\left(h_{1}-h_{1_{0}}\right)^{2}+\cdots+\left(h_{n}-h_{n_{0}}\right)^{2}+w\left(Q_{i}-Q_{0}\right)^{2}$,

where $w$ is the weight of the defined seepage flow. Thus, the adaptability value of each frog can be obtained.

(d) These frogs are ranked in descending order according to their adaptability values after mixing into the frog population; then, these frogs are constantly improved from procedure (B) based on the mentioned method in the 2.4 algorithm model.

(e) When either ending requirement in 2.3 is met, the programming will be ended and the best frogs can be obtained. These frogs are the final result of inverse analysis of the seepage parameters; thus, the seepage condition of the dam can be calculated by the finite element model.

\section{Comparison with the Results of Sand Sink Experiment}

A certain homogeneous isotropic rectangular dam has two horizontal drainage layers. The size of the rectangular dam is $0.55 \mathrm{~m} \times 0.35 \mathrm{~m} \times 0.1 \mathrm{~m}$ (length $\times$ height $\times$ width), and its seepage parameters is $k_{1}$. The size of the horizontal drainage layer is $0.35 \mathrm{~m} \times 0.04 \mathrm{~m} \times 0.1 \mathrm{~m}$ (length $\times$ height $\times$ width), and its seepage parameter is $k_{2}$. The detail sizes and arrangements of the sand sink experiment model are shown in Figure 2, in which the unit is $\mathrm{cm}$. The upstream water of the rectangular dam is $0.35 \mathrm{~m}$, and there is no water downstream. The experimental liquid is soluble glass [21].

The rectangular coordinate system is shown in Figure 2, in which the lower left corner point of the sand sink experiment model is chosen as coordinate O. According to the rectangular dam prototype of the sand sink experiment, the finite element model, including the 


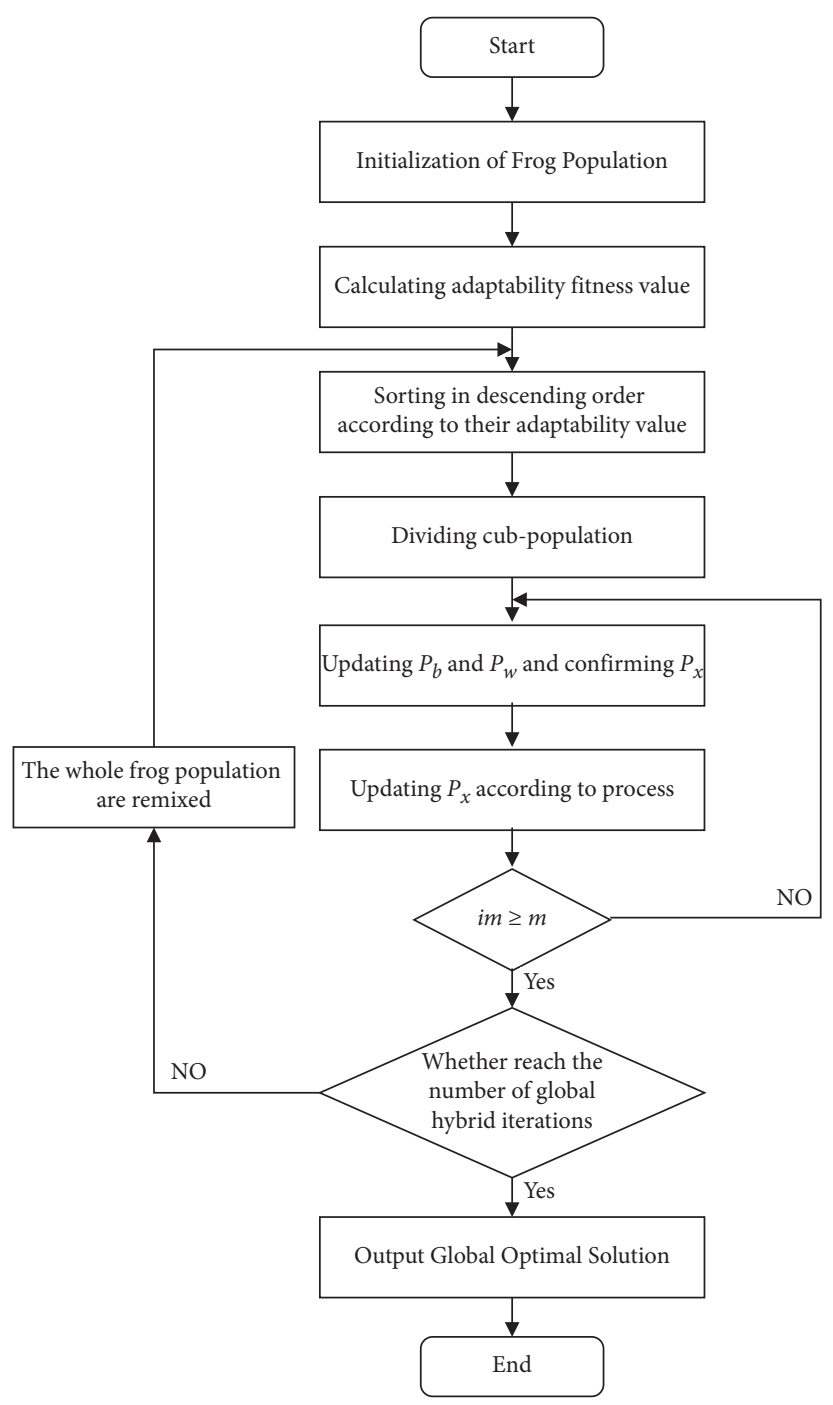

Figure 1: Flowchart of SFLA.

TABle 1: Calculation results of test function.

\begin{tabular}{lccc}
\hline Function & Optimization algorithm & Average optimal solution & Theoretical optimal solution \\
\hline \multirow{2}{*}{ Sphere } & SFLA & $1.236 \mathrm{E}-3$ & 0 \\
& ISFLA & 0.000 & 0 \\
\multirow{2}{*}{ Rosenbrock } & SFLA & 2.549 & 0 \\
& ISFLA & $8.424 \mathrm{E}-3$ & 0 \\
\multirow{2}{*}{ Griewangk } & SFLA & $2.710 \mathrm{E}-1$ & 0 \\
& ISFLA & $1.587 \mathrm{E}-2$ & 0 \\
\multirow{2}{*}{ Schaffer's f7 } & SFLA & 4.994 & $2.102 \mathrm{E}-1$ \\
\hline
\end{tabular}

horizontal drainage layers, is established for seepage analysis. The finite element model is shown in Figure 3. There are 800 nodes and 744 elements in this model. The boundary conditions of the model are as follows: (1) there is a waterhead of $0.35 \mathrm{~m}$ on the upstream surface, which is the determined hydraulic head boundary, (2) there is no water on the downstream surface, which is the seepage boundary, (3) the underside of the model is the impervious boundary. There are two materials in this experiment model: one is the body of a rectangular dam and the other is the horizontal drainage layers.

Considering the drainage effect of the horizontal drainage layers, the free surface position of the observation points are $A(15.0,27.43), B(25.0,17.85), C(35.0,16.17)$, and $\mathrm{D}(45.0,9.25)$ in the sand sink experiment. Meanwhile, the stable seepage flow is $5.35 \times 10^{-5} \mathrm{~m}^{2} / \mathrm{s}$. 


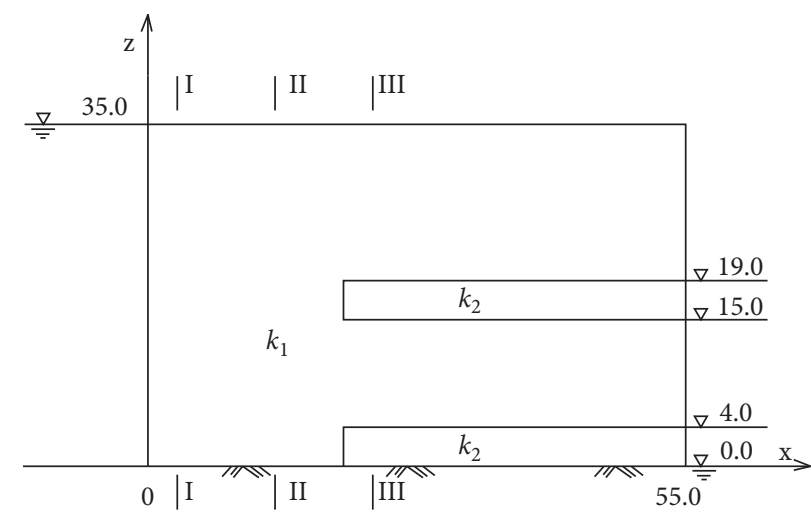

FiguRE 2: Sand sink experiment model of the homogeneous isotropic rectangular dam (unit: $\mathrm{cm}$ ).

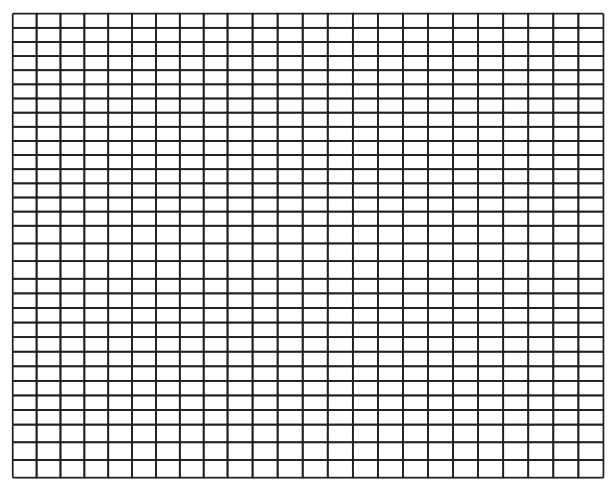

FIGURE 3: Finite element model of homogeneous rectangular dam.

According to the results of sand sink experiment, the objective function of the free surface position and the seepage flow is established, which is the square sum of the difference between the value of these experiment results above and the inverse seepage characteristics In this objection, the inverse seepage characteristics are calculated by the finite element method based on the seepage parameters of the dam $k_{1}$ and the horizontal drainage layers $k_{2}$, which are the variable values that will finally be determined by the objective function.

The finite element program for seepage inverse analysis based on ISFLA, which has the accuracy control during the calculation, has chosen 10-time iterative analysis as the number of times for the effective evaluation control. Set the local update threshold $R_{0}=0.6$; the number of frogs in one memeplex $n=39$ (1 less than the actual value); the number of times of the local memetic evolution $L S=15$; the number of times of the global thoughts exchange $S F=10$; and other parameters are the same as in chapter 3. The running environment is Microsoft visual c++ system, Intel (R) Core (TM) i5-4200 CPU, 2 cores, 4 GB RAM. The results of the inverse analysis, including the seepage parameters of the dam, the horizontal drainage layers, and the difference are obtained based on ISFLA; meanwhile, the runtime of inverse iteration calculation for each time is recorded. The results of the inverse analysis for sand sink experiment are shown in Table 2.
The seepage parameter of the rectangular dam $k_{1}$ is $2.21 \times 10^{-4} \mathrm{~m} / \mathrm{s}$ and the horizontal drainage layer $k_{2}$ is $3.50 \times 10^{-3} \mathrm{~m} / \mathrm{s}$, which are measured by the sand sink experiment. Meanwhile, the calculated result of the inverse analysis to the seepage parameters of the rectangular dam $k_{1}$ is $2.21 \times 10^{-4} \mathrm{~m} / \mathrm{s}$ and the horizontal drainage layer $k_{2}$ is $3.53 \times 10^{-3} \mathrm{~m} / \mathrm{s}$ based on ISFLA. The free surface and the distribution of the seepage contour line are shown in Figure 4. Compared with the results between the inverse analysis and the experiment, it can be seen that there is little difference between $k_{1}$ and $k_{2}$, which are merely $0.05 \%$ and $0.86 \%$ between the experiment and inverse analysis. It is shown that ISFLA can be applied to do the seepage inverse analysis and obtain the seepage parameters; what is more, it is also proven that ISFLA has excellent precision and good global optimization capability. It takes $4468 \mathrm{~s}$ for the calculation of the inverse analysis with overall 10 times, which means that each iteration inverse analysis only needs $446.8 \mathrm{~s}$ on average. It is can also be seen that, under the local threshold $R_{0}=0.6$, ISFLA has excellent efficiency for the inverse analysis of seepage parameters. Furthermore, choosing 5 8 times of the iteration inverse analysis when the ISFLA is applied to the inversion analysis of seepage parameters because the result can also get good accuracy can be proposed. 
TABLE 2: The results of the inverse analysis of the sand sink experiment.

\begin{tabular}{|c|c|c|c|c|}
\hline Inverse analysis times & $\mathrm{Dam} / 10^{-4} \mathrm{~m} / \mathrm{s}$ & Drainage layer $/ 10^{-3} \mathrm{~m} / \mathrm{s}$ & Objective function $/ 10^{-4}$ & Running time (s) \\
\hline 1 & 2.20 & 3.63 & 71 & 860 \\
\hline 2 & 2.21 & 3.50 & 0 & 508 \\
\hline 3 & 2.21 & 3.48 & 6 & 267 \\
\hline 4 & 2.21 & 3.50 & 0 & 265 \\
\hline 5 & 2.21 & 3.50 & 0 & 426 \\
\hline 6 & 2.21 & 3.51 & 4 & 259 \\
\hline 7 & 2.21 & 3.52 & 7 & 405 \\
\hline 8 & 2.21 & 3.49 & 3 & 186 \\
\hline 9 & 2.20 & 3.63 & 71 & 641 \\
\hline 10 & 2.22 & 3.52 & 26 & 651 \\
\hline Average & 2.21 & 3.53 & 19 & 446.8 \\
\hline
\end{tabular}

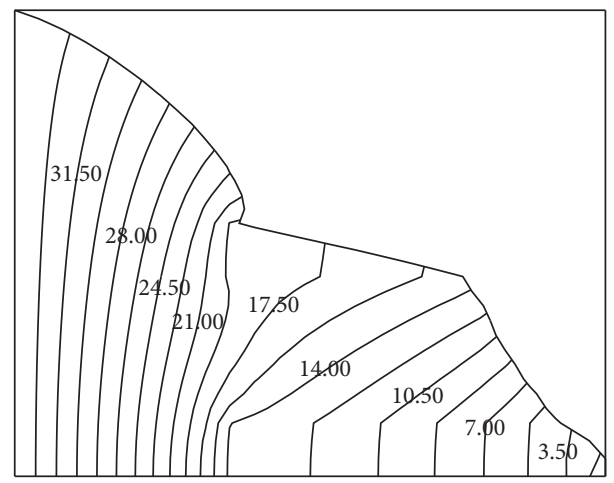

FIgURE 4: Free surface and the distribution of the seepage contour line based on ISFLA.

\section{Engineering Application of Inverse Analysis of Seepage Parameters Based on ISFLA}

A medium-sized reservoir, located in Lianyungang city, Jiangsu province, China, is used for flood control and irrigation. The reservoir's design flood standard is 50 years and the check flood standard is 1000 years. The design water level is $49.23 \mathrm{~m}$ and the check water level is $50.04 \mathrm{~m}$. The reservoir capacity of the reservoir is $21,110,000 \mathrm{~m}^{3}$. The waterretaining structure of the reservoir is a clay homogeneous earth dam. The length of the dam is $1850 \mathrm{~m}$ and the crest width of the dam is 5.5 to $6.0 \mathrm{~m}$. The elevation of the dam is $52.0 \mathrm{~m}$, and the maximum height of the dam is $15.0 \mathrm{~m}$. The typical section of the dam is shown in Figure 5. This reservoir started to operation in 1958.

The dam has been reinforced during operation, but some sections of the dam in the back-towards-water slope still suffer from the problems of leakage, which lead to the dam toe being swamp-like. Therefore, to figure out the seepage characteristics of sick dangerous dams after running for years, as well as providing the theoretical basis for the reinforce method of the dam, it is necessary to promote the research on seepage inverse analysis to seepage parameters of the dams and the foundations.

According to the report of geological investigation, the experiment of the soil sampling by drilling, and the standard penetration test, the lithology of the dam can be obtained. The dam and its foundation are mainly comprised of (1) filling clay in the dam, (4) sand layer, (5) completely weathered gneiss, (6) strongly weathered gneiss, and (7) moderately weathered gneiss. According to the laboratory tests, on-site water injection test, the suggested seepage parameters of the above materials, shown in Table 3, can be obtained based on comprehensive analysis. The groundwater level in drillings by on-site observation is shown in Table 4. In the period of the drillings survey, the upstream water level is $45.59 \mathrm{~m}$ and the downstream is $40.5 \mathrm{~m}$.

It is necessary to know the rationality of the suggested seepage parameters in Table 3. ISFLA should be applied for inverse analysis to seepage parameters based on the groundwater level in drillings by on-site observation; thus, the seepage characteristics of the dam can be obtained, as well as the seepage parameters of the dam and the foundation. The soil layers which will greatly affect the seepage characteristics of the dam should be chosen for inverse analysis. Therefore, according to the information of engineering geological investigation, there are two soil layers, (1) filling clay in the dam and (4) sand layer, which are chosen for the inverse analysis of the seepage parameters.

According to the dam material partition of the dam shown in Figure 5, the finite element model for seepage analysis is established including the dam body and its foundation. The rectangular coordinate system is combined by $\mathrm{X}$ and $\mathrm{Y}$. $\mathrm{X}$ is the horizontal direction that is vertical to the dam axis and the coordinate origin of the $\mathrm{X}$-axis is on the upstream dam crest, while $\mathrm{Y}$ is the vertical direction that is the direction of actual elevation. The range of the model is 


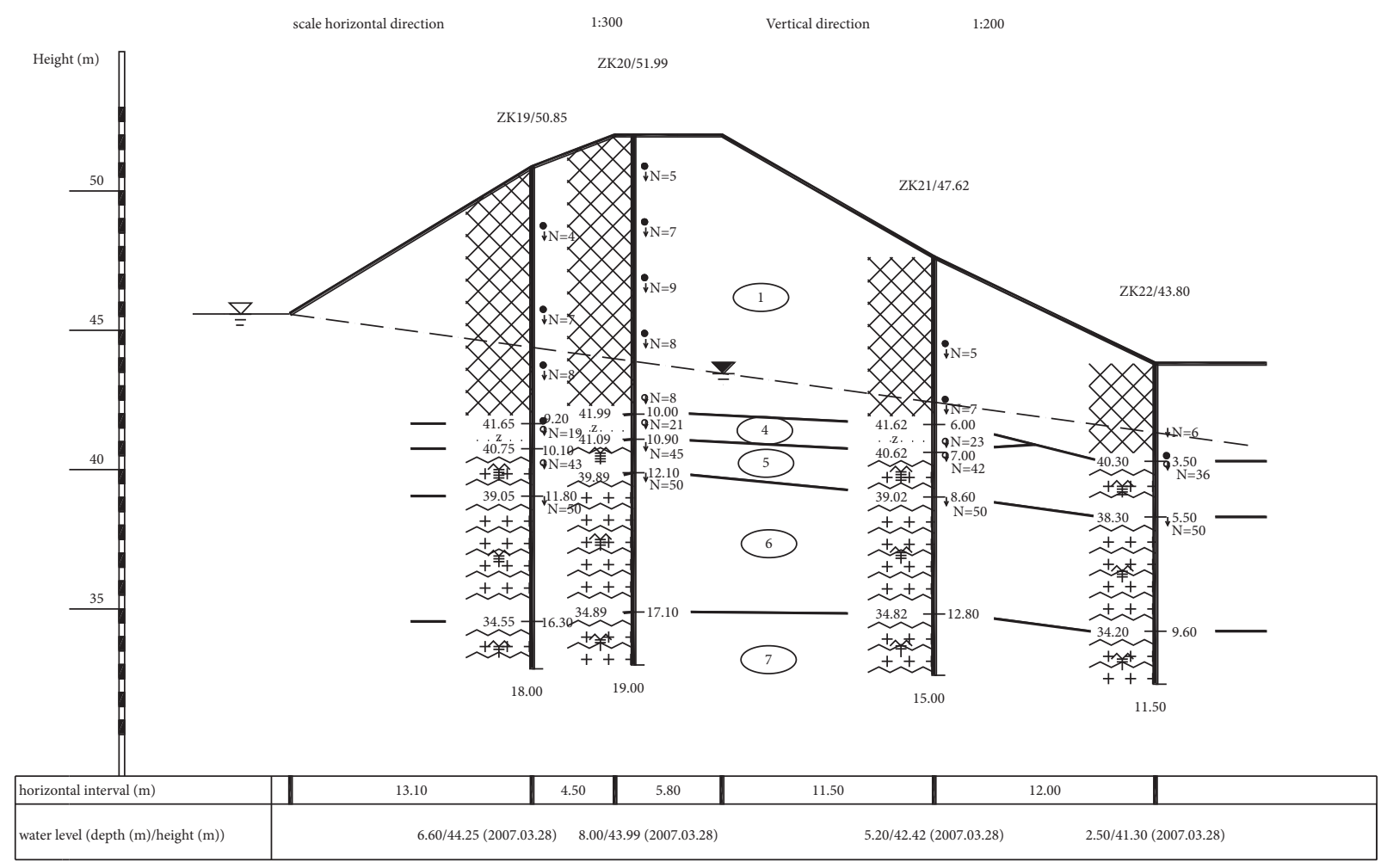

FIgURE 5: Typical section of the dam.

chosen as follows: the left boundary of the model is $27.0 \mathrm{~m}$ away from the dam heel (about twice the height of the dam), the right boundary of the model is $27.0 \mathrm{~m}$ away from the dam toe (about twice the height of the dam), and the bottom boundary of the model is $22.5 \mathrm{~m}$ away from the dam foundation surface (about 1.5 times the height of the dam). The finite element model is shown in Figure 6. There are 2382 nodes and 2297 elements in this model. The boundary conditions of the model are as follows: (1) the determined hydraulic head boundary, including the surface of the upstream dam slope and reservoir bottom under the upstream water level (the upstream water level is $45.59 \mathrm{~m}$ ), the surface of the downstream dam slope and reservoir bottom under the downstream water level (the downstream water level is $40.5 \mathrm{~m}),(2)$ the seepage boundary, including the surface of upstream dam slope above upstream, the surface of downstream dam slope above downstream water level and the surface of the dam crest, (3) the impervious boundary, including the surface of the left side, right side, and the underside of the model.

The objective function, the square sum of the difference between the value of groundwater level in drillings by on-site observation and the calculated results of the free surface by ISFLA, is established. It is chosen 10 times for the iteration in this inverse analysis of the seepage parameters. The ISFLA model parameters and the running environment are the same as in chapter 5 . Based on the above theoretic, the seepage parameters of (1) filling clay in the dam and (4) sand layer, the square sum of the difference, and the time used in each inverse iteration are all obtained, which are listed in Table 5.
According to Table 5, the average results of the seepage parameters of (1) filling clay in the dam and (4) sand layer are $2.33 \times 10^{-7} \mathrm{~m} / \mathrm{s}, 9.30 \times 10^{-6} \mathrm{~m} / \mathrm{s}$. Compared with the suggested values of them, both of the calculated results are decreased, which are reduced by $29.8 \%$ and $62.5 \%$, respectively. Meanwhile, the permeability coefficients of the filling clay in the dam have the same order of magnitude as the suggested value. In addition, it only costs $6921 \mathrm{~s}$ in total for 10 times iteration inverse analysis, which means that there is only $692.1 \mathrm{~s}$ for each time of inverse analysis on average. Therefore, it is can also be shown that ISFLA has excellent efficiency for the inverse analysis of seepage parameters when it is applied to engineering. Based on the average results of the seepage parameters, the seepage characteristics of the dam can be obtained. Thus, the comparison between the value of groundwater level in drillings by on-site observation and the finite element method is shown in Table 6. The free surface and the distribution of the seepage contour line are shown in Figure 7, where there are the dashed lines.

From Table 6, considering the damming height $H=5.09 \mathrm{~m}$, it can be proven that the relative error between calculated results and on-site observation values is small, the maximum of which is only $7.66 \%$. It is also proved that seepage characteristics of the dam can be well calculated by the inverse analysis results of the seepage parameters.

Furthermore, the inverse analysis of seepage parameters is applied to study the seepage characteristics of the dam under the normal water level; meanwhile, the rationality of the inverse analysis of seepage parameters can be proven once again. The conditions of the normal water level are as 
TABLE 3: Suggested seepage parameters of the dam and foundation.

\begin{tabular}{lcc}
\hline Soil layer numbers & Soil layer names & Suggested values $(\mathrm{m} / \mathrm{s})$ \\
\hline 1 & Filling clay & $3.32 \times 10^{-7}$ \\
4 & Sand layer & $2.48 \times 10^{-5}$ \\
5 & Completely weathered gneiss & $3.42 \times 10^{-6}$ \\
6 & Strongly weathered gneiss & $4.36 \times 10^{-7}$ \\
7 & Moderately weathered gneiss & $5.45 \times 10^{-8}$ \\
\hline
\end{tabular}

TABLE 4: Groundwater level in drillings by on-site observation.

\begin{tabular}{lllcr}
\hline Drillings numbers & ZK19 & ZK20 & ZK21 & ZK22 \\
\hline Water level $(\mathrm{m})$ & 44.25 & 43.99 & 42.42 & 41.3 \\
\hline
\end{tabular}

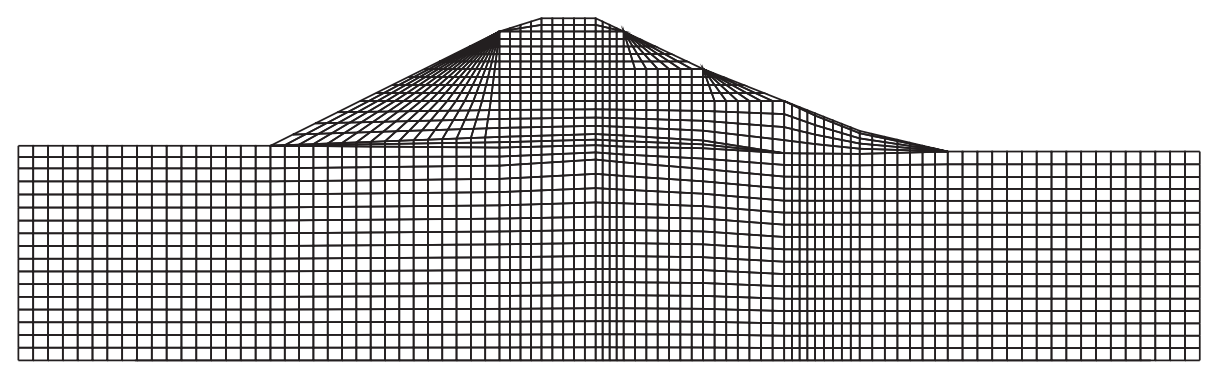

FIGURE 6: Finite element model of the dam.

TABLE 5: Inverse analysis results of the seepage parameters.

\begin{tabular}{lcccc}
\hline Inverse analysis times & Filling clay in the dam $/ 10^{-7} \mathrm{~m} / \mathrm{s}$ & Foundation sand layer $/ 10^{-6} \mathrm{~m} / \mathrm{s}$ & Objective function & Running time $(\mathrm{s})$ \\
\hline 1 & 1.87 & 9.42 & 0.648 & 6.645 \\
2 & 1.89 & 9.48 & 0.625 & 679 \\
3 & 2.52 & 8.31 & 0.634 & 735 \\
4 & 2.91 & 9.80 & 0.595 & 707 \\
5 & 1.78 & 9.13 & 0.644 & 706 \\
6 & 2.25 & 8.64 & 0.565 & 680 \\
7 & 2.18 & 9.74 & 0.645 & 693 \\
8 & 1.90 & 9.51 & 0.642 & 684 \\
9 & 3.00 & 9.26 & 0.597 & 681 \\
10 & 3.03 & 9.72 & 0.624 & 697 \\
Average & 2.33 & 9.30 & 692.1 \\
\hline
\end{tabular}

TABLE 6: Comparison between the value of groundwater level in drillings by on-site observation and the finite element method.

\begin{tabular}{lcccc}
\hline Drillings numbers & X coordinate $(\mathrm{m})$ & Calculated results $(\mathrm{m})$ & On-site observation values $(\mathrm{m})$ & Relative error $(\%)$ \\
\hline ZK19 & -4.50 & 43.96 & 44.25 & 5.70 \\
ZK20 & 1.00 & 43.65 & 43.99 & 6.68 \\
ZK21 & 17.35 & 42.72 & 42.42 & 5.89 \\
ZK22 & 29.35 & 41.69 & 41.30 & 7.66 \\
\hline
\end{tabular}

follows: the upstream normal water level is $48.5 \mathrm{~m}$, and there is no water downstream. The free surface and the distribution of the seepage contour line are shown in Figure 7 , in which it is the solid lines. There is no leakage at the downstream dam toe when the upstream normal water level is $45.59 \mathrm{~m}$; however, the free surface exits from the downstream dam toe when the upstream normal water level is
$48.5 \mathrm{~m}$. These results are in keeping with the actual situation that the dam is under the problems of leakage at the backtowards-water slope. Thus, it can be proven that the inverse analysis of the seepage parameters has good accuracy, and the calculated seepage characteristics of the dam can be considered as the real state of dam's seepage. What is more, the permeability coefficients of the sand layer in the dam 


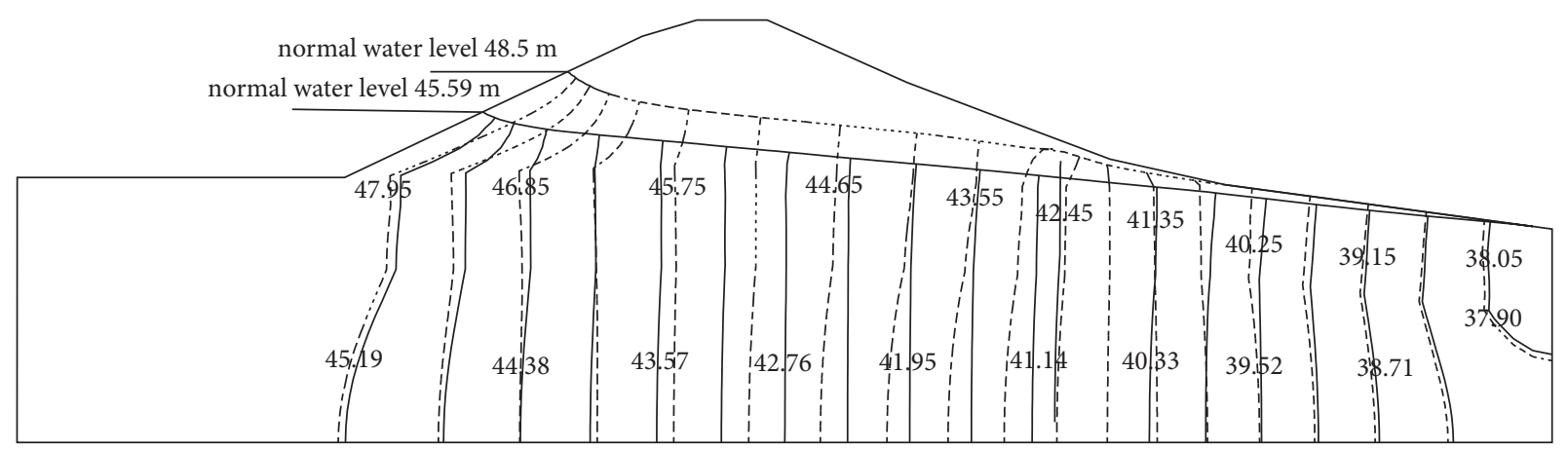

FIGURE 7: Free surface and distribution of the seepage contour line.

foundation are large, which means that the dam foundation has the leakage problem, so it is necessary to reinforce the foundation to solve the problem of being swamp-like at the back-towards-water slope and meet the requirement of seepage stability.

\section{Conclusions}

ISFLA is an efficient and practical numerical method to search for the optimal solution, such as some of the most representative computational intelligence algorithms such as the monarch butterfly optimization (MBO) [22], earthworm optimization algorithm (EWA) [23], and elephant herding optimization algorithm (EHO) [24]. By combining ISFLA and the finite element method for seepage, the program for inverse analysis of seepage parameters is completed. The accuracy and efficiency of the program are proven by a comparative example; moreover, it is applied to solve the problem of engineering in Lianyungang, China. Based on the above results, the conclusions are as follows:

(1) Based on the basic theory of SFLA, considering the seepage characteristics, such as the seepage flow and the value of the waterhead as the objective function, the inverse analysis of seepage parameters is proposed by ISFLA.

(2) According to the seepage parameters results from the sand sink experiment and inverse analysis calculation by ISFLA, it is proven that ISFLA has excellent precision, excellent efficiency, and good global optimization capability. Furthermore, when ISFLA is applied to the inversion analysis of the seepage parameter, it can be proposed that $5 \sim 8$ times of the iteration inverse analysis can meet the requirement of the precision.

(3) According to the leakage problem in the clay dam in Lianyungang, China, the inverse analysis of seepage parameters to the earth dam are studied by the ISFLA and the finite element method; meanwhile, the seepage characteristics of the dam can be obtained. Moreover, the relative error between the calculated results and on-site observation values is obtained. It is proven that ISFLA has excellent efficiency for the inverse analysis of seepage parameters when it is applied to engineering. Finally, the suggestion that the dam should be reinforced is put forward.

\section{Data Availability}

The data used to support the findings of this study are available from the corresponding author upon request.

\section{Conflicts of Interest}

The authors declare that they have no conflicts of interest.

\section{Acknowledgments}

This work was funded by the Project of National Natural Science Foundation of China/Yalong River Joint Fund (Grant no. U1765205) and the National Key R\&D Program of China (Grant no. 2019YFC1510802).

\section{References}

[1] X. Q. Niu, "Characteristics of reservoir defects and rehabilitation technology in China," Chinese Journal of Geotechnical Engineering, vol. 32, no. 1, pp. 153-157, 2010.

[2] Z. R. Wu, Safety Monitor Theory \& its Application of Hydraulic Structures, Higher Education Press, Beijing, China, 2003.

[3] Y. F. Chen, C. B. Zhou, and R. Hu, "Key issues on seepage flow analysis in large scale hydropower projects," Chinese Journal of Geotechnical Engineering, vol. 32, no. 9, pp. 1448-1454, 2010.

[4] D. Y. Li, X. Q. Gan, and W. Zhou, "Back analysis on mechanical parameters of dams based on uniform design and genetic neural network," Chinese Journal of Geotechnical Engineering, vol. 29, no. 1, pp. 125-130, 2007.

[5] L. H. Yao, "Parameters identification of groundwater flow model with genetic algorithm and gauss-Newton method," Chinese Journal of Geotechnical Engineering, vol. 27, no. 8, pp. 885-890, 2005.

[6] Y. Wang and J. Liu, "Back analysis of the permeability coefficients of gravity dam foundation by evolution method," Chinese Journal of Geotechnical Engineering, vol. 25, no. 5, pp. 552-556, 2003.

[7] J. Liu and Y. Wang, "Improved genetic algorithm in back analysis for seepage parameters of fissured rock masses," Rock and Soil Mechanics, vol. 24, no. 2, pp. 237-241, 2003.

[8] Y. Song, D. Wu, W. Deng et al., "Mppcede: multi-population parallel co-evolutionary differential evolution for parameter 
optimization," Energy Conversion and Management, vol. 228, no. 2, Article ID 113661, 2021.

[9] W. Deng, J. Xu, Y. Song, and H. Zhao, "Differential evolution algorithm with wavelet basis function and optimal mutation strategy for complex optimization problem," Applied Soft Computing, vol. 100, Article ID 106724, 2020.

[10] W. Deng, J. Xu, H. Zhao, and Y. Song, "A novel gate resource allocation method using improved PSO-based QEA," IEEE Transactions on Intelligent Transportation Systems, pp. 1-9, 2020.

[11] M. M. Eusuff and K. E. Lansey, "Optimization of water distribution network design using the shuffled frog leaping algorithm," Journal of Water Resources Planning and Management, vol. 129, no. 3, pp. 210-225, 2003.

[12] Y. H. Li, J. Z. Zhou, J. J. Yang, and L. Liu, "An improved hybrid leapfrog algorithm based on threshold selection strategy," Computer Engineering and Application, vol. 43, no. 35, pp. 19-21, 2007.

[13] E. Elbeltagi, T. Hegazy, and D. Grierson, "Comparison among five evolutionary-based optimization algorithms," Advanced Engineering Informatics, vol. 19, no. 1, pp. 43-53, 2005.

[14] A. Rahimi-Vahed and A. H. Mirzaei, "A hybrid multi-objective shuffled frog-leaping algorithm for a mixed-model assembly line sequencing problem," Computers \& Industrial Engineering, vol. 53, no. 4, pp. 642-666, 2007.

[15] G. Chung and K. Lansey, "Application of the shuffled frog leaping algorithm for the optimization of a general large-scale water supply system," Water Resources Management, vol. 23, no. 4, pp. 797-823, 2009.

[16] H. Elbehairy, E. Elbeltagi, T. Hegazy, and K. Soudki, "Comparison of two evolutionary algorithms for optimization of bridgedeck repairs," Computer-Aided Civil and Infrastructure Engineering, vol. 21, no. 8, pp. 561-572, 2006.

[17] J. P. Luo, X. Li, and M. R. Chen, "Improved shuffled frog leaping algorithm for solving CVRP," Journal of Electronics \& Information Technology, vol. 33, no. 2, pp. 429-434, 2011.

[18] Y. Han, J. H. Cai, G. G. Zhou, Y. L. Li, H. Z. Lin, and J. F. Tang, "Advances in shuffled frog leaping algorithm," Computer Science, vol. 37, no. 7, pp. 16-19, 2010.

[19] G. Y. Zhu and W. Q. Lin, "Mounting sequential optimization on surface mounting machine using improved hybrid frogjumping algorithm," Chinese Journal of Construction Machinery, vol. 6, no. 4, pp. 428-432, 2008.

[20] Z. Y. Xuan and C. J. Zhang, "A shuffled frog leaping algorithm for solving knapsack problem," Science Technology and Engineering, vol. 9, no. 15, pp. 4363-4365, 2009.

[21] Z. M. Yang, "Study on seepage control optimization problem of the tailings dam," Master's thesis, Hohai University, Nanjing, China, 1992.

[22] G. G. Wang, S. Deb, Z. Cui, M. Lu, and X. Zhao, "Monarch butterfly optimization," Neural Computing and Applications, vol. 31, no. 7, pp. 1995-2014, 2019.

[23] G. G. Wang, S. Deb, and L. D. S. Coelho, "Earthworm optimisation algorithm: a bio-inspired metaheuristic algorithm for global optimisation problems," International Journal of Bio-Inspired Computation, vol. 12, no. 1, pp. 1-22, 2015.

[24] G. G. Wang, L. Dos Santos Coelho, X. Z. Gao, and S. Deb, “A new metaheuristic optimisation algorithm motivated by elephant herding behaviour," International Journal of Bio-Inspired Computation, vol. 8, no. 6, pp. 394-409, 2016. 